\title{
Disorders of Consciousness
}

\author{
Caterina Pistarini and Giorgio Maggioni
}

\section{Introduction}

Consciousness is defined as the state of awareness of the self and environment with appropriate arousal or wakefulness (Giacino et al. 2018a, b). Disorders of Consciousness (DoC) are a wide spectrum of correlates of brain's disruptions of arousal and awareness that may result from altered functional neural activities from cortico-cortical connectivity to subcortico-cortical and global connectivity of all networks such as default mode network (DMN) and others (Giacino et al. 2014; Hodelìn-Tablada 2016).

The mentioned neural networks that define consciousness are primarily assessed by clinical means (i.e., scale like CRS-r-Coma Recovery Scale revised) to detect patient's behavior and capability to have and show conscious experience; these are the only validated means, while some critics recently arose (Bayne et al. 2017; Seel et al. 2010).

Arousal is clinically assessed by evidence of eye opening and brain stem reflexes and is defined by a spectrum of conditions from sleep to complete wakefulness.

Awareness may be clinically evaluated by the examination of motor and communication behaviors assessing visual pursuit, localization to noxious stimulation, command following, intelligible verbalization, and object recognition in order to define the perceiving of the external environment and the voluntary interaction with it (Laureys et al. 2004).

\footnotetext{
C. Pistarini

Department of NeuroRehabilitation Medicine,

ICS Maugeri SB, Institute of Genova Nervi, Genoa, GE, Italy

e-mail: caterina.pistarini@icsmaugeri.it

G. Maggioni $(\bowtie)$

NeuroRehabilitation Unit, Department of NeuroRehabilitation Medicine,

IRCCS ICS Maugeri Veruno SB, Institute of Veruno, Gattico-Veruno, NO, Italy

e-mail: giorgio.maggioni@icsmaugeri.it
} 
Perturbations of arousal and awareness depict different clinical syndromes of DoC including coma, different sleep stages, drug anesthesia, vegetative state, minimally conscious state, and emerging state (Blume et al. 2015).

Coma, defined by the absence of arousal and consciousness, sleep stages and drug-induced alterations are not at center of our interest while we refer to the other mentioned syndromes on a rehabilitation point of view.

DoC are defined on the basis of assessment of motor and communication behaviors; a coma lasting more than 28 days may lead to different DoC, starting from vegetative state (VS) or otherwise named "unresponsive wakeful syndrome" (UWS): in this case, patients are unaware but awake showing eye opening (both spontaneously or induced) and reflexive movements (Giacino et al. 2018a, b; Laureys et al. 2010).

In case the VS (UWS) clinical condition will last more than 1 month, it should be classified as "persistent" and not as "permanent."

When patients show voluntary behavior, usually beginning with visual pursuit, they are in the clinical condition of the minimally conscious state (MCS) with inconsistent but reproducible behavior demonstrating awareness of external or internal world (Giacino et al. 2014).

Recently, the complexity of behavioral responses allows to classify MCS patients in "plus or minus" (MCS -/MCS +): in the first case, patients show interactions with external environment only through motor behavior and in the latter also some preserved language function. Any functional object use or evidence of accurate and functional communication will define the emerging state from MCS (EMCS) (Bruno et al. 2011; Giacino et al. 2014).

We refer to Table 1 and to the cited articles for detailed description of DoC conditions.

Table 1 Different features of clinical presentation of DoC (adapted from: Eapen et al. 2017; Giacino et al. 2014)

\begin{tabular}{l|l|l|l|l|l}
\hline & $\begin{array}{l}\text { Sleep-wake } \\
\text { cycle }\end{array}$ & Arousal & Awareness & $\begin{array}{l}\text { Motor } \\
\text { purposeful } \\
\text { behavior }\end{array}$ & $\begin{array}{l}\text { Preserved language } \\
\text { function }^{\text {b }}\end{array}$ \\
\hline Coma & Absent & Absent & Absent & $\begin{array}{l}\text { Absent/ } \\
\text { reflexives }\end{array}$ & Absent \\
\hline VS/UWS & $\begin{array}{l}\text { Present, } \\
\text { altered, } \\
\text { intermittent }\end{array}$ & Present & Absent & $\begin{array}{l}\text { Absent/ } \\
\text { reflexives }\end{array}$ & Absent \\
\hline MCS- & Present & Present & Present & Present & Absent \\
\hline MCS+ & Present & Present & Present & Present & Present \\
\hline $\begin{array}{l}\text { Emerging } \\
\text { state }\end{array}$ & Present & Present & Present & Present & $\begin{array}{l}\text { Present: Reliable } \\
\text { communication; use of } \\
\text { functional object }\end{array}$ \\
\hline
\end{tabular}

${ }^{a}$ Motor purposeful behavior: visual pursuit, localization to noxious stimulation, simple command following

bPreserved language function: intelligible verbalization, object recognition, command following 
While the definition and our understanding of the pathomechanisms of DoC improved in recent years, we are still facing the difficulty of correct diagnosis and related procedures such as appropriate assessment of awareness and alertness and partially as a consequence have limited valid evidence regarding beneficial treatment options including rehabilitation or medication (Bender et al. 2015; Bodien et al. 2017).

Recent data on the annual incidence of VS/UWS in the US is 4200 persons/year with reported prevalence rates ranging from 5000 to 42,000 (112,000-280,000 for MCS); while prevalence on a world basis of UWS/VS is $0.2-6.1 / 100,000$ inhabitants (Giacino et al. 2018a, b; Van Erp et al. 2014).

The repeatedly cited $42 \%$ rate of misdiagnosis is now decreasing to a $37 \%$ minimum but has yet to be precisely defined (Bender et al. 2015; Peterson et al. 2015; Wade 2018).

In fact, the proper means and clinical algorithm to perform a correct diagnosis of UWS/VS and MCS are still a matter of debate (Giacino et al. 2013).

Nontraumatic coma (NTC) is primarily caused by stroke (6-54\%) when compared to post-anoxic or metabolic injuries; but it is still difficult to describe DoC while the patients is in the acute hospital ward and only the presence of a coma lasting more than 28 days may depict the consciousness impairment as a steady disorder such as UWS/VS or MCS and not as a transient event (Woimant et al. 2014).

The available literature relates to either traumatic (TBI) or nontraumatic (nTBI) DoC mainly. Few studies consider cerebrovascular accidents (CVA) specifically and fewer discriminate cerebral infarction, parenchymal hemorrhage, and subarachnoidal bleeding; a recent systematic review could not describe more precisely DoC in stroke alone (Horsting et al. 2015).

Evidence is lacking regarding diagnosis and natural history in nTBI MCS patients while four studies with nTBI VS patients note a survival rate of $80 \%$ on average (95\% confidence interval, 95\% CI "67\% to 93\%") at 3 months (Hannawi et al. 2015; Horsting et al. 2015; Kondziella et al. 2016; O’Donnel et al. 2019). Little is known about any differentiation between CVA, cardiac arrest, and metabolic DoC.

While a systematic approach to the rehabilitation of DoC exists since almost 30 years, many problems related to clinical diagnosing, assessments, and treatment remain unsolved (De Tanti et al. 2015; Kondziella et al. 2016; Giacino et al. 2018a, b). As one example, the use of the reliable and valid assessment tools is essential to properly define the clinical pattern, to plan the care process adequately, and ensure a precise prognosis (Di Perri et al. 2014; Eapen et al. 2017; Giacino et al. 2014).

This chapter reviews the available evidences regarding DoC after a stroke and as a whole.

Topics for our evidence synthesis and practice recommendations are: (1) DoC assessment including technical diagnostic tools and clinical assessments; (2) general DoC rehabilitation; (3) specific DoC treatment options including drug therapy, technology-based treatment, music therapy, surgical therapies, and noninvasive brain stimulation (NIBS). 


\section{$2 \quad$ Methods}

We systematically searched for guidelines, reviews, and clinical trials that provide evidence and clinical decision guidance for DoC assessment including technical diagnostic tools and clinical assessments; general DoC rehabilitation; or specific DoC treatment options. Exclusion criteria were: non-English papers, commentaries, case series, case reports, book chapters, and conference reports.

We, in January 2019, searched for articles published from 1997 to January 2019 in the PubMed, Cochrane Library using medical subject heading (MeSH) keywords and the search algorithm "(rehabilitation OR stroke OR cerebral hemorrhage OR cerebral infarction) AND (disorders of consciousness OR persistent vegetative state OR vegetative state OR minimally conscious state)," and in the EMBASE via the EMTREE with following keywords and algorithm "(Cerebrovascular accident) AND (disorders of consciousness OR persistent vegetative state OR vegetative state OR minimally conscious state)."

We found a total of 862 articles: two independent raters screened the 862 articles, solving disagreement by discussion.

Systematic reviews $(n=11)$, nonsystematic reviews $(n=13), \operatorname{RCT}(N=2)$, observational studies $(N=12)$ were selected together with two guidelines for full-text review to critically appraise and collate the evidence, to rate the overall quality of evidences, and to provide recommendations.

Two clinical evidence and practice guidelines (GL) were found (De Tanti et al. 2015; Giacino et al. 2018a, b).

The level of evidence used for recommendations was categorized according to the Oxford Centre for Evidence-Based Medicine Levels of Evidence (CEBM, 2009 version).

Further, the quality of the evidence was rated with four categories according to "GRADE" ("Grades of Recommendation, Assessment, Development, and Evaluation”) (Owens et al. 2010):

- High quality: further research is unlikely to affect our confidence in the estimation of the (therapeutic) effect;

- Medium quality: further research is likely to affect our confidence in the estimation of the (therapeutic) effect and may alter the estimate;

- Low quality: further research will most likely influence our confidence in the estimation of the (therapeutic) effect and will probably change the estimate;

- Very low quality: any estimation of the (therapy) effect or prognosis is very uncertain.

- The grading of the recommendations was performed in accordance with GRADE with the categories "ought to" (A) (strong recommendation), "should" (B) (weak recommendation) (Schünemann et al. 2013). As a third category had been introduced "can" (0) (option) (Platz 2017). 


\section{DoC Assessment: Clinical Behavioral and Instrumental Diagnostic Tools}

\subsection{Clinicals and Behavioral Tools for DoC Assessments}

A systematic review of the main assessment behavioral means for clinical practice identified 13 DoC scales and reported on their inter-rater reliability (IRR), internal consistency (IC), and test-retest reliability (TRR) qualities (Seel et al. 2010).

Authors of this review also studied other features of assessment scales such as existent guidelines for their application, their power to detect DoC, i.e., VS and MCS, to differentiate between the two syndromes and their feasibility to be used in clinical practice. Considered scales were: the Coma Recovery Scale-revised (CRSr), Sensory Stimulation Assessment Measure (SSAM), Wessex Head Injury Matrix (WHIM), Western Neuro Sensory Stimulation Profile (WNSSP), Sensory Modality Assessment Technique (SMART), Disorders of Consciousness Scale (DoC), Coma/ Near-Coma Scale (CNC), Full Outline of UnResponsiveness Score (FOUR), Comprehensive Levels of Consciousness Scale (CLOCS), Innsbruck Coma Scale (INNS), Glasgow-Liege Coma Scale (GLS), Loewenstein Communication Scale (LOEW), and the Swedish Reaction Level Scale-1985 (RLS85).

CRS-r was considered superior to the other scales while review states that many unproven features still remain; in Table 2 are summarized diagnostic features of all mentioned scales.

Overall diagnostic validity of the assessment scales was unproven; in particular, the prognostic and the diagnostic validity of CRS-r are not proven. The only scale with a good predictive power for good recovery at 30 days was FOUR (Wolf et al. 2007). Systematic review conducted to define the American Academy of Neurology guidelines found little evidences with no recommendation possible: a study considered 43 patients and found CRS-r as an independent predictor of recovery with wide CIs limit (95\% CI "1.05 to 11643.58") of interpretation (Estraneo et al. 2013; Luauté et al. 2010).

Recommendation:

Clinical assessment of DoC, i.e., VS and MCS should be performed with standardized assessment tools in patients with prolonged DoC (CEBM classification: level 1a, GRADE moderate; recommendation B+). The CRS-r appears to have advantages concerning its psychometric properties; its use is suggested (CEBM classification: level 1a, GRADE moderate; recommendation $B+$ ).

\subsection{Instrumental Diagnostic Tools for DoC Assessment}

One systematic review (SR), following established recommendations for conducting SR including Preferred Reporting Items for Systematic review and MetaAnalysis protocols (PRISMA) and checklist for SR such as the Quality Assessment 


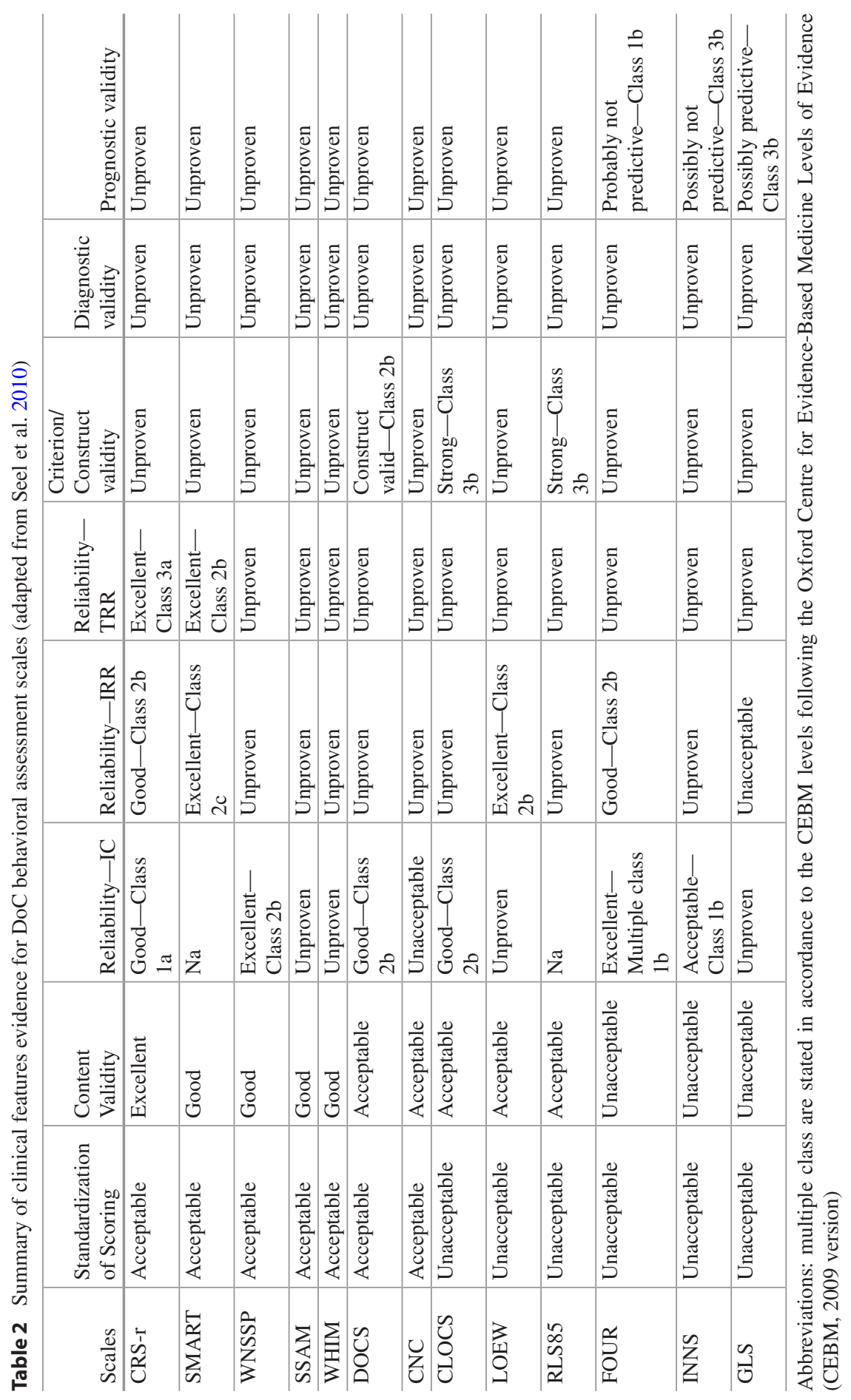


of Diagnostic Accuracy Studies-2 (QUADAS-2), analyzed the usefulness of neurophysiological recordings to assess DoC patients, focusing on electroencephalography (EEG), electromyography (EMG), Event-related Potentials (ERP), and Mismatch Negativity (MMN) (Hauger et al. 2017; Kable et al. 2012; Mother et al. 2015).

Results in this SR are not conclusive: specificity rates of different methods were highly variable from $0 \%$ to $100 \%$, with a high rate of false-positive classifications. Two studies report a rate of $17 \%$ and $33 \%$ of false-positive classifications of UWS instead of MCS (King et al. 2013; Sitt et al. 2014).

An SR and meta-analysis considered 20 clinical studies with a total sample of 470 MCS patients and 436 VS patients studied with quantitative method EEG (qEEG), ERP, functional magnetic resonance imaging (fMRI): qEEG showed the highest sensitivity (90\% on average; $95 \%$ CI "69\% to 97\%") and high specificity ( $80 \%$ on average with $95 \%$ CI " $66 \%$ to $90 \%$ ") compared to ERP and fMRI; the sensitivity of fMRI was $44 \%$ on average (95\% "CI $19 \%$ to $72 \%$ ") and specificity $67 \%$ on average (95\% CI " $55 \%$ to $77 \%$ "), while the sensitivity of ERP was $59 \%$ on average (95\% CI "26\% to 85\%") and specificity $75 \%$ on average (95\% CI "51\% to 90\%”) (Bender et al. 2015).

A third SR included 36 studies with 687 patients $(10.3 \%$ with ischemic stroke and $6.6 \%$ with intracerebral hemorrhage and $2.7 \%$ with subarachnoid hemorrhage) studied with fMRI, fluorodeoxyglucose (FDG)-positron emission tomography (PET), single-photon emission computed tomography (SPECT) with different tracers (Hannawi et al. 2015). These approaches could detect reduced activity in several brain areas and may be useful in clinical DoC diagnosis, in particular considering modifications of the Default Mode Network (DMN); but the heterogeneity observed in study results and the meta-analysis conducted in 13 studies (272 patients and 259 controls) showed that the small number of studies that reported increased functional connectivity limited the importance of results with little support in clinical practice (Hannawi et al. 2015).

An SR included 1041 patients from 44 originals articles (54.1\% VS and $45.9 \%$ MCS; $25 \%$ of all DoC patients had CVA) to study EEG, fMRI (ERP were excluded in this SR) to detect consciousness with active and passive paradigms such as the cortical functional connectivity or resting-state studies, and on same sample of patients a meta-analysis was performed on 37 studies: researchers failed to indicate a diagnostic superiority for the analysis of active and passive paradigms considering both command-no command following and cortical-no cortical connectivity on an fMRI motor imagery task to distinguish MCS and VS compared to EEG: Odds ratio (OR) of EEG versus fMRI were 0.73 (95\% CI " 0.50 to 1.07 ") in the active paradigms and 1.78 (95\% CI " 1.16 to 2.74 ") in the passive paradigms; the authors concluded that EEG protocols were two times more likely to be interpreted as compatible with preserved consciousness compared to fMRI (Kondziella et al. 2016).

A study on EEG mainly showed that MCS patients had less abnormal EEG findings $(61 \%, 95 \%$ CI " $41 \%$ to $78 \%$ ") compared to VS patients $(25 \%, 95 \%$ CI " $7 \%$ to $59 \%$ ") with a sensitivity for MCS diagnosis of $61 \%$ (95\% CI "39\% to $80 \%$ ") (Casarotto et al. 2016). 
There is, however, no evidence available to support EEG as a diagnostic tool to distinguishing VS from MCS on an individual basis.

There is insufficient evidence that electromyography (EMG) may support the diagnostic value of the presence of EMG activity to a command: a study considered 38 DoC patients and in only one VS patient and three MCS patients an EMG response to command was found (sensitivity for MCS 21\%, 95\% CI "5\% to 51\%", and specificity $90 \% 95 \%$ CI "56\% to 100\%") (Habbal et al. 2014).

In conclusion, the accuracy in diagnostic approach of neurophysiological and other technical tools is limited. (1) EEG evaluation seems to be the best method, with good balance between rates for sensitivity and specificity; (2) ERP may help to detect reactivity to few sensory stimuli; (3) fMRI may be useful to specifically study the activity of different functional networks; and (4) EMG may be only of little help to distinguish MCS from VS patients with reference to voluntary movements.

\section{Recommendation:}

Assessment of DOC with qEEG can be performed in patients with prolonged $D O C$ as a supplementary diagnostic approach (CEBM classification: level 1, GRADE moderate; recommendation 0). For all other approaches, i.e., EMG, ERP, and fMRI the evidence is too weak to give a recommendation for their routine diagnostic use in prolonged DOCs (CEBM classification: level 1, GRADE moderate).

\section{DoC Rehabilitation}

The complex approach of rehabilitation for DoC patients, though it is highly recommended, is difficult to appraise critically. There are only very few randomized controlled trials (RCT) available.

RCTs are of course difficult to perform in DoC patients because of the control groups required, long-lasting follow-up, little acceptance to participate while caregivers are easily reluctant to consent to trials limiting treatment options if not as "last option" that might induce a lack of valid samples for studies (Giacino et al. 2013).

A small number of studies have evaluated issues related to the early rehabilitation phase of DoC (Pistarini and Maggioni 2018). Seel made, based on a singlecenter retrospective study, six recommendations for a better ER management but little evidences arise (Seel et al. 2013). Authors studied data on CRS-r scale scores pre-post rehabilitation and discharge disposition in a sample of 210 patients with DoC (VS and MCS) and 53\% of patients showed at least one or more signs of emergence into full consciousness (26\% emerged from MCS in 2 CRS-r criteria and 27\% in 1 of CRS-r criteria (Seel et al. 2013).

Rehabilitation methods used range from stretching exercises, postures (early mobilization/verticalization), splinting, casting, range of motion mobilization, but no RCT studied them. Only case studies, observational studies, and nonsystematic reviews are available (Giacino et al. 2013).

Previous systematic and nonsystematic reviews stated that structured sensory stimulation could not positively be recommended, if it was to be based on relevant clinical evidence (Georgiopoulos et al. 2010; Lombardi et al. 2002). 
One systematic review retrieved 157 articles with 18 studies investigating MCS/ VS patients with spasticity (Martens et al. 2017). Three prospective studies reported an occurrence of spasticity in DoC patients. Six articles focused only on spasticity treatments as primary outcome: 2 were clinical trials, 2 case reports, and last 2 were reviews. The rehabilitation methods studied were splinting and acupuncture, but no outcome differences were elicited when comparing splinting to physiotherapy sessions, and the hypothesis of decreasing overexcitability in muscle hypertonia induced by acupuncture is not yet validated (Thibaut et al. 2015).

Available treatments for spasticity range from the use of drugs such as phenol, botulinum toxin, baclofen, tizanidine, dantrolene sodium, diazepam to physical treatments, in particular passive range of motion and stretching, splinting orthoses, casting, positioning, to surgical approaches such as intrathecal baclofen therapy and selective posterior rhizotomy. All available studies show little evidences of efficacy with a low-GRADE quality scoring. Other nine reports (2 clinical trials, 1 openlabel study, 4 case reports, and 2 reviews) observed and studied spasticity treatments as secondary outcome, including deep brain stimulation to induce Cortical Activation by Thalamic Stimulation (CATS) and intrathecal baclofen (ITB) pump: no RCT among all selected studies are existing (Magrassi et al. 2016; Margetis et al. 2014). All studies analyzed could not depict any evidence for differential treatment effects.

While no evidence is available to support specific forms of rehabilitation treatment in DoC patients, the experience from clinical practice indicates that an interdisciplinary rehabilitation approach that addresses the various therapeutic needs of DoC patients should be stated as early as possible and continued for a reasonable time to promote functional recovery including recovery of arousal and awareness as well as of emerging motor and communication functions.

At a later stage, such interdisciplinary rehabilitation treatment might be repeated to reevaluate the potential for functional recovery.

Recommendation:

Complex interdisciplinary rehabilitation should be provided by a team experienced with patients with prolonged DOCs, started in an early phase, as soon as the clinical condition allows, with an individualized approach (CEBM classification: level 5, GRADE quality: very low [expert opinion], recommendation grade: $B+$ ). It should be continued for a reasonable time to ensure that a potential for gradual recovery has a chance to evolve under specialized treatment and might be repeated at later stages to reevaluate the potential for recovery (CEBM classification: level 5, GRADE quality: very low [expert opinion], recommendation grade: $B+$ ).

\section{$5 \quad$ Pharmacological Therapies for DoC}

Several drugs have been used in the therapy of patients with DoCs. Most frequently used were centrally acting drugs including dopamine and GABA agonists. Among the first mentioned class are bromocriptine, levodopa, and apomorphine. Most frequently used GABA agonists are zolpidem and baclofen (both oral and intrathecal-ITB). Among most used drugs are then amantadine and methylphenidate. 
Studies on dopamine agonists either have considerable methodological restrictions or used very small samples; they often do not consider effects of spontaneous recovery and/or do not describe correctly clinical conditions with reference to comorbidities: neither results nor any suggestions may then be reported from those studies (Gosseries et al. 2014).

Only studies on amantadine report results that provide sufficient evidence for clinical decision-making. Specifically, an RCT compared the effects of a prescription of amantadine versus placebo over a treatment course of 4 weeks in $184 \mathrm{DoC}$ (only traumatic DoC in VS-MCS) patients (Giacino et al. 2012).

The outcomes were disability measured with the Disability Rating Score scale (DRS) and clinical DOC assessment with the CRS-r.

Results showed a significantly faster recovery in the treatment group (mean difference 0.24 DRS point per week $95 \%$ CI " $0.07 \%$ to $0.4 \%$ "). At 2 weeks, after washout, the difference decreased while remaining significant and at 6 weeks follow-up, the DRS scores were similar between control and treatment group with no significant difference (mean difference-0.7 DRS point per week 95\% CI " -2 to 10.7"). No significant differences in result arose when considering the analysis of CRS-r.

Data available on zolpidem are conflicting. In a non-randomized, placebocontrolled trial only one out of $15 \mathrm{DoC}$ (12 VS and $3 \mathrm{MCS}$ ) patient showed improvement in CRS-r scoring, being able to open her eyes sustainably (Whyte and Myers 2009).

All other studies reviewed are not of high quality (no RCT); while it has been reported in at least 6 studies and 23 clinical reports that zolpidem may induce EEG activation, may enhance BOLD signal in different brain regions, and activate metabolicandneuronalactivity studied withPETscans, M-EEG,Magnetoencephalography, and MRI imaging, a clinically relevant beneficial therapeutic effect of zolpidem on DOC had not been shown with an appropriate clinical trial to address this question (Gosseries et al. 2014).

No RCTs are available on ITB: few clinical reports support that the use of ITB, mainly for spasticity treatment purposes, may facilitate recovery; these articles are mainly clinical reports on a small sample and do not provide sufficient evidence for clinical decision-making process (Francois et al. 2001; Shrestha et al. 2011; Thibaut et al. 2015).

Recommendation.

A course of amantadine treatment over a couple of weeks can be used in the beginning of the rehabilitation treatment of stroke survivors with DoC (VS-MCS) to promote recovery in the disability domain (CEBM classification: level 1b, GRADE quality: moderate, recommendation grade: 0 [indirectness of evidence]).

The evidence is too limited to guide clinical decision-making with respect to long-term use and discontinuation of amantadine, or the prescription of other drugs to treat DoC in stroke survivors. 


\section{Other Specific Therapies for DoC}

One RCT enrolled 44 out of 50 initially considered patients with DoC (14 VS and $30 \mathrm{MCS}$ ) and analyzed the results from the use of a tilt table therapy compared to tilt therapy integrated with a stepping device (product Erigo ${ }^{\circledR}$ by Hocoma, Switzerland); both groups received $1 \mathrm{~h}$ sessions over a course of 3 weeks (Krewer et al. 2015).

The primary outcome was the improvement rate of CRS-r scores after treatment, and the secondary outcome was the difference in the Modified Ashworth Scale (MAS) again after treatment. Both control and treatment group improved, and the intervention effect for the stepping device was less when changes scores from baseline to week 6 were analyzed (median $\left(25 \%-75 \%\right.$ percentile): Erigo ${ }^{\circledR} 4(-1-6)$; tilt table: 9 (5-10); $U$-Test $=122.0 ; z=-2.824, p=0.005, r=-0.42)$. Changes in MAS scoring did not significantly differ between the two patient groups.

A case series investigated the effects on CRS-r scoring and electrophysiological criteria in response to the use of deep brain stimulation (DBS) in $21 \mathrm{VS}$ patients with an increase odd of recovery (OR 88.0; 95\% CI "5.4 to 1219.0") (Yamamoto et al. 2010). No control group was considered and the sample was very small.

Other specific therapies used to treat DoC that were investigated are music therapy, noninvasive brain stimulation (NIBS) such as $20 \mathrm{~Hz}$ repetitive transcranial magnetic stimulation (rTMS) and transcranial direct current stimulation (tDCS) (Bai et al. 2017; He et al. 2018; Rollnik and Altenmuller 2014).

Only clinical studies on a small sample basis are available. Few considerations are reported in nonsystematic reviews like the targeting of the left dorsolateral prefrontal cortex in DoC when using tDCS or rTMS, but no evidence that would be sufficient for clinical decision-making process (Hodelìn-Tablada 2016; Pignat et al. 2015).

Recommendation:

The evidence is too limited to guide clinical decision-making for therapies such as tilt therapy with integrated stepping device, rTMS, or tDCS when used with the intention to treat DoC in stroke survivors. Their use is discouraged for routine clinical practice with the therapeutic goal to improve DoC in stroke survivors (CEBM classification: level 4 to $1 \mathrm{~b}, \mathrm{GRADE}$ quality: very low to moderate, recommendation grade: $B-)$.

\section{References}

Bai Y, Xia X, Kang J, Yang Y, He J, Li X (2017) TDCS modulates cortical excitability in patients with disorders of consciousness. NeuroImage Clin 15:702-709

Bayne T, How J, Owen AM (2017) Reforming the taxonomy in disorders of consciousness. Ann Neurol 82(6):866-872 
Bender A, Jox RJ, Straube A, Lulé D (2015) Persistent vegetative state and minimally conscious state. A systematic review and meta-analysis of diagnostic procedures. Dtsch Arztebl Int 112:235-242

Blume C, del Giudice R, Wislowska M, Lechinger J, Schabus M (2015) Across the consciousness continuum-from unresponsive wakefulness to sleep. Front Hum Neurosci 9:105

Bodien YG, Chatelle C, Edlow BL (2017) Functional networks in disorders of consciousness. Semin Neurol 37(5):485-502

Bruno MA, Vanhaudenhuyse TA, Moonen G, Laureys S (2011) From unresponsive wakefulness to minimally conscious PLUS and functional locked-in syndromes: recent advances in our understanding of disorders of consciousness. J Neurol 258(7):1373-1384

Casarotto S, Comaducci A, Rosanova M (2016) Stratification of unresponsive patients by an independently validated index of brain complexity. Ann Neurol 80:718-729

De Tanti A, Zampolini M, Pregno S, on behalf of the CC3 Group (2015) Recommendations for clinical practice and research in severe brain injury in intensive rehabilitation: the Italian consensus conference. Eur J Rehabil Med 51:89-103

Di Perri C, Stender J, Laureys S, Gosseries O (2014) Functional neuroanatomy of disorders of consciousness. Epilepsy Behav 30:28-32

Eapen BC, Georgekutty J, Subbarao B, Bavishi S, Cifu DX (2017) Disorders of consciousness. Phys Med Rehabil Clin N Am 28:245-258

Estraneo A, Moretta P, Loreto V, Lanzillo B, Cozzolino A, Saltalamacchia A, Lullo F, Santoro L, Trojano L (2013) Predictors of recovery of responsiveness in prolonged anoxic vegetative state. Neurology 80:464-470

Francois B, Vacher P, Roustan J, Salle JY, Vidal J, Moreau JJ, Vignon P (2001) Intrathecal baclofen after traumatic brain injury: early treatment using a new technique to prevent spasticity. $\mathrm{J}$ Trauma 50:158-161

Georgiopoulos M, Katsakiori P, Kafalopoulou Z, Ellul J, Chroni E, Constantoyannis C (2010) Vegetative state and minimally conscious state: a review of the therapeutic interventions. Stereotact Funct Neurosurg 88:199-207

Giacino JT, Whyte J, Bagiella E, Kalmar K, Child N, Khademi A, Eifert B, Long D, Katz DI, Cho S, Yablon SA, Luther M, Hammond FM, Nordenbo A, Novak P, Mercer W, Maurer-Karattup S, Sherer M (2012) Placebo controlled trial of amantadine for severe traumatic brain injury. N Engl J Med 366:819-826

Giacino JT, Katz DI, Whyte J (2013) Neurorehabilitation in disorders of consciousness. Semin Neurol 33:142-156

Giacino JT, Fins JJ, Laureys S, Schiff ND (2014) Disorders of consciousness after acquired brain injury: the state of the science. Nat Rev Neurol 10(2):99-114

Giacino JT, Katz DI, Schiff ND, Whyte J, Ashman EJ, Ashwal S, Barbano R, Hammond F, Laureys S, Ling GSF, Nakase-Richardson R, Seel RT, Yabloon S, Getchius TSD, Gronseth GS, Armstrong MJ (2018a) Practice guideline update recommendations summary: disorders of consciousness. Neurology 91(10):450-460

Giacino JT, Katz DI, Schiff ND, Whyte J, Ashman EJ, Ashwal S, Barbano R, Hammond F, Laureys S, Ling GSF, Nakase-Richardson R, Seel RT, Yabloon S, Getchius TSD, Gronseth GS, Armstrong MJ (2018b) Comprehensive systematic review update summary: disorders of consciousness. Arch Phys Med Rehabil 99:1710-1719

Gosseries O, Charland-Verville V, Thonnard M, Bodard O, Laureys S, Demertzi D (2014) Amantadine, Apomorphine and Zolpidem in the treatment of disorders of consciousness. Curr Pharm Des 20:4167-4184

Habbal D, Gosseries O, Noirhomme Q (2014) Volitional electromyografic responses in disorders of consciousness. Brain Inj 28:1171-1179

Hannawi Y, Lindquist MA, Caffo BS, Sair HI, Stevens RD (2015) Resting brain activity in disorders of consciousness. A systematic review and meta-analysis. Neurology 84:1272-1280

Hauger SL, Schanke A-K, Andersson S, Chatelle C, Schnakers C, Løvstad M (2017) The clinical diagnostic utility of electrophysiological techniques in assessment of patients with disorders 
of consciousness following acquired brain injury: a systematic review. J Head Trauma Rehabil 32(3): 185-196

He F, Wu M, Meng F, Hu Y, Gao J, Chen Z, Bao W, Liu K, Luo B, Pan G (2018) Effects of 20 Hz repetitive transcranial magnetic stimulation on disorders of consciousness: a resting-state electroencephalography study. Neural Plast 25:5036184

Hodelìn-Tablada R (2016) Minimally conscious state: evolution of concept, diagnosis and treatment. MEDICC Rev 18(4):43-46

Horsting MWB, Franken MD, Meulenbelt J, van Klei WA, de Lange DW (2015) The etiology and outcome of non-traumatic coma in critical care: a systematic review. BMC Anesthesiol 15:65

Kable AK, Pich J, Maslin-Prothero SE (2012) A structured approach to documenting a search strategy for publication: a 12-step guideline for authors. Nurse Educ Today 32(8):878-886

King J, Faugeras F, Gramfort A (2013) Single-trial decoding of auditory novelty responses facilitates the detection of residual consciousness. Neuroimage 83:726-738

Kondziella D, Friberg CK, Frokjaer VG, Fabricius M, Møller K (2016) Preserved consciousness in vegetative and minimal conscious states: systematic review and meta-analysis. J Neurol Neurosurg Psychiatry 87:485-492

Krewer C, Luther M, Koenig E, Müller F (2015) Tilt table therapies for patients with severe disorders of consciousness: a randomized, controlled trial. PLoS One 10(12):e0143180

Laureys S, Owen AM, Schiff ND (2004) Brain function in coma, vegetative state, and related disorders. Lancet Neurol 3(9):537-546

Laureys S, Celesia GG, Cohadon F, Lavrijsen J, Léon-Carrion J, Sanita WG, Sazbon L, Schmutzhard E, von Wil KR, Zeman A, Dolce G (2010) Unresponsive wakefulness syndrome: a new name for the vegetative state or apallic syndrome. BMC Med 8:68

Lombardi F, Taricco M, De Tanti A, Telaro E, Liberati A (2002) Sensory stimulation for brain injured individuals in coma or vegetative state. Cochrane Database Syst Rev 2002(2):CD001427

Luauté J, Maucort-Boulch D, Tell L (2010) Long-term outcomes of chronic minimally conscious and vegetative state. Neurology 75:246-252

Magrassi L, Maggioni G, Pistarini C, Di Perri C, Bastianelle S, Zippo AG, Iotti GA, Biella GE, Imberti R (2016) Results of a prospective study (CATS) on effects of talami stimulation in minimally conscious and vegetative state patients. J Neurosurg 125(4):972-981

Margetis K, Korfias SI, Gatzonis S, Boutos N, Stranjalis G, Boviatsis E, Sakas DE (2014) Intrathecal baclofen associated with improvements of consciousness disorders in spasticity patients. Neuromodulation 17:699-704

Martens G, Laureys S, Thibaut A (2017) Spasticity management in disorders of consciousness. Brain Sci 7(12):E162

Mother D, Shamseer L, Clarke M (2015) Preferred reporting items for systematic review and metaanalysis protocols (PRISMA-P). Syst Rev 4:1

Owens D, Lohr K, Atkins D, Treadwell JR, Reston JT, Bass EB, Chang S, Helfand M (2010) AHRQ Series Paper 5: Grading the strength of a body of evidence when comparing medical interventions: AHRQ and the Effective Health Care Program. J Clin Epidemiol 63: $513-523$

O'Donnel JC, Browne KD, Kilbaugh TJ, Chen HI, Whyte J, Cullen DK (2019) Challenges and demand for modeling disorders of consciousness following traumatic brain injury. Neurosci Biobehav Rev 98:336-346

Peterson A, Cruse D, Naci L, Weijer C, Owen AM (2015) Risk, diagnostic error, and the clinical science of consciousness. NeuroImage Clin 7:588-597

Pignat JM, Johr J, Diserens K (2015) From disorders of consciousness to early Neurorehabilitation using assistive technologies in patients with severe brain injury. Curr Opin Neurol 28:587-594

Pistarini C, Maggioni G (2018) Early rehabilitation od disorders of consciousness (DOC): management, neuropsychological evaluation and treatment. Neuropsychol Rehabil 28(8): $1319-1330$

Platz T (2017) Practice guidelines in neurorehabilitation. Neurol Int Open 01(03):E148-E152. https://doi.org/10.1055/s-0043-103057 
Rollnik JD, Altenmuller E (2014) Music in disorders of consciousness. Front Neurosci 8:190

Schünemann H, Brożek J, Guyatt G, Oxman A (2013) GRADE handbook for grading quality of evidence and strength of recommendations. The GRADE Working Group. Updated October 2013. www.guidelinedevelopment.org/handbook

Seel TR, Sherer M, Whyte J, Katz DI, Giacino JT, Rosenbaum AM, Hammond FM, Kalmar K, Pape TLB, Zafonte R, Biester RC, Kaelin D, Kean J, Zaslr N (2010) Assessment scales for disorders of consciousness: evidence-based recommendations for clinical practice and research. Arch Phys Med Rehabil 91(12):1795-1813

Seel TR, Douglas J, Dennison AC, Heaner S, Harris K, Rogers C (2013) Specialized early treatment for persons with disorders of consciousness: program components and outcomes. Arch Phys Med Rehabil 94(10):1908-1923

Shrestha P, Malla H, Pant B, Taira T (2011) Intrathecal baclofen therapy in severe brain injury, first time in Nepal, a technique suitable for underdeveloped countries. Asian J Neurosurg 6:49-51

Sitt JD, King JR, Karoui IEI (2014) Large scale screening of neural signature of consciousness in patients in a vegetative state or minimally conscious state. Brain J Neurol 137(8):2258-2270

Thibaut A, Deltombe T, Wannez S, Gosseries O, Ziegler E, Dieni C, Deroy M, Laureys S (2015) Impact of soft splints on upper limb spasticity in chronic patients with disorders of consciousness: a randomized single-blind, controller trial. Brain Inj 29:830-836

Van Erp WS, Lavrijsen JCM, van de Laar FA, Vos PE, Laureys S, Koopmans RTCM (2014) The vegetative state/unresponsive wakefulness syndrome: a systematic review of prevalence studies. Eur J Neurol 21(11):1361-1368

Wade DT (2018) How often is the diagnosis of the permanent vegetative state incorrect? A review of the evidence. Eur J Neurol 25:619-625

Whyte J, Myers R (2009) Incidence of clinically significant responses to zolpidem among patients with disorders of consciousness: a preliminary placebo-controlled trial. Am J Phys Med Rehabil 88(5):410-418

Woimant F, Biteye Y, Chaine P, Crozier S (2014) Severe stroke: which medicine for which results? Annales Francaises d'Anesthésie et de Réanimation 33:102-109

Wolf CA, Wijdicks EFM, Bamlet WR, McClelland RL (2007) Further validation of the FOUR score coma scale by intensive care nurses. Mayo Clin Proc 82:435-438

Yamamoto T, Katayama Y, Kobayashi K, Oshima H, Fukaya C, Tsubokawa T (2010) Deep brain stimulation for the treatment of vegetative state. Eur J Neurosci 32(7):1145-1151

Open Access This chapter is licensed under the terms of the Creative Commons AttributionNonCommercial-NoDerivatives 4.0 International License (http://creativecommons.org/ licenses/by-nc-nd/4.0/), which permits any noncommercial use, sharing, distribution and reproduction in any medium or format, as long as you give appropriate credit to the original author(s) and the source, provide a link to the Creative Commons license and indicate if you modified the licensed material. You do not have permission under this license to share adapted material derived from this chapter or parts of it.

The images or other third party material in this chapter are included in the chapter's Creative Commons license, unless indicated otherwise in a credit line to the material. If material is not included in the chapter's Creative Commons license and your intended use is not permitted by statutory regulation or exceeds the permitted use, you will need to obtain permission directly from the copyright holder. 